\title{
"Reconstructions of the past distribution of Testudo graeca mitochondrial lineages in the Middle East and Transcaucasia support multiple refugia since the Last Glacial Maximum": A response to Turkozan et al. (2021)
}

\author{
Flora Ihlow ${ }^{1}$, Uwe Fritz ${ }^{1}$, Peter Mikulíček ${ }^{2}$ \& Dennis Rödder ${ }^{3}$ \\ ${ }^{1}$ Museum of Zoology Senckenberg Dresden, Königsbrücker Landstraße 159, 01109 Dresden, Germany
${ }^{2}$ Department of Zoology, Faculty of Natural Sciences, Comenius University in Bratislava, Ilkovičova 6, 84215 Bratislava, Slovakia
${ }^{3}$ Zoologisches Forschungsmuseum Alexander Koenig (ZFMK), Adenauerallee 160, 53113 Bonn, Germany
}

Species distribution models (SDMs) are frequently used to characterise current, past or future realised environmental niches. Two recent studies applied different approaches to infer range dynamics in eastern subspecies of the spur-thighed tortoise Testudo graeca Linnaeus, 1758. We discuss differences in the conclusions of the two papers and use multivariate environmental similarity surface (MESS) analyses to show that the results of the study by Turkozan et al. (2021), recently published in the Herpetological Journal, are compromised by extrapolation and therefore have to be interpreted with caution.

Keywords: Glacial refugia, multivariate environmental similarity surface (MESS), range shifts, species distribution modelling, spur-thighed tortoise

U nderstanding how endangered taxa are distributed is a basic prerequisite for conservation planning and, in the face of the sixth mass extinction event during Earth's history affecting vertebrates (cf. Ceballos et al., 2020), of paramount importance. Ever refined approaches for species distribution modelling substantially contribute to a better knowledge of the current, past and future distribution ranges of chelonians (e.g., Ihlow et al., 2012; Rödder et al., 2013), one of the most threatened vertebrate groups (TTWG, 2017). Recently, Turkozan et al. (2021) aimed to clarify the distribution and past range dynamics of spur-thighed tortoises (Testudo graeca) harbouring different mitochondrial lineages that are generally identified as distinct subspecies (TTWG, 2017). We appreciate the efforts undertaken by Turkozan et al. (2021) but found some misconceptions in their article that we highlight in this note.

Turkozan et al. (2021) used species distribution models (SDMs) to predict the ranges of the five eastern subspecies of $T$. graeca. SDMs are frequently applied to characterise current realised environmental niches and estimate potential geographic distributions of taxa. By projecting SDMs onto paleoclimatic or putative future conditions, range shifts can be inferred. However, predictor variables are extrapolated when projecting models through space (whenever the projection area is larger than the training range) and time (projecting onto future or past climatic conditions; e.g., Elith et al., $2009,2010)$. This requires cautious interpretation of modelling results (Elith et al., 2010; Owens et al., 2013). Extrapolation effects tend to increase when models are trained with geographically restricted data sets (e.g., Elith et al., 2010; Rocchini et al., 2011; Engler \& Rödder, 2012; Owens et al., 2013).

Turkozan et al. (2021) inferred environmental niche models for each of the five studied subspecies using ten uncorrelated bioclimatic predictors (seven temperaturerelated and three precipitation-related variables) and the maximum entropy modelling algorithm MaxEnt (Phillips et al., 2006; Phillips \& Dudík, 2008). Another recent study (Javanbakht et al., 2017) examined three of these subspecies using $n$-dimensional hypervolumes based on principal components derived from 19 bioclimatic variables (cf. Blonder et al., 2014; Blonder, 2018). In order to study paleoclimatic range dynamics, both teams projected their resulting models onto reconstructions of climatic conditions of the mid-Holocene $(6,000 \mathrm{BP})$ and the Last Glacial Maximum (LGM, 21,000 BP) but came to different conclusions.

According to Turkozan et al. (2021), the distribution ranges of two subspecies (T.g. armeniaca and T.g. buxtoni) were almost exclusively shaped by a single precipitationrelated variable, respectively (with permutation contributions of $95.8 \%$ and $85 \%$ ), while the ranges of $T$. g. ibera and T. g. zarudnyi were predominantly affected by a single temperature-related predictor (75\% and 88 $\%$, respectively). The distribution of $T$. g. terrestris was inferred to be shaped by precipitation- and temperaturerelated predictors. In contrast, Javanbakht et al. (2017) found that the distribution of T. g. armeniaca, T. $g$. buxtoni, and T. g. zarudnyi was predominantly limited 
by precipitation. For the latter subspecies, this conflicts with the results of Turkozan et al. (2021).

Turkozan et al. (2021) suggested that multiple glacial refugia existed and that since the LGM the potentially suitable geographic space has expanded for $T$. $g$. ibera, contracted for $T$. g. zarudnyi, and remained stable for $T$. g. terrestris. For T. g. armeniaca and T. g. buxtoni, the patterns were inconclusive. In contrast, Javanbakht et al. (2017) suggested that the ranges of the three studied subspecies (T. g. armeniaca, T. g. buxtoni, T. g. zarudnyi) experienced only slight shifts and did not expand significantly after the LGM.

The methods used by Turkozan et al. (2021) and Javanbakht et al. (2017) are fundamentally different, having distinct underlying conceptual and computational principles. Correlative SDMs, such as MaxEnt used by Turkozan et al. (2021), are prone to extrapolation errors when projected through space and time (Elith et al., 2010; Owens et al., 2013). In contrast, profiling techniques based on multivariate analyses, such as the non-parametric kernel density estimation (KDE) and $n$-dimensional hypervolumes used by Javanbakht et al. (2017), are more robust and allow the characterisation of realised niches based on delimitation of niche volumes. Especially when calibration areas are small (VanDerWal et al., 2009), projections onto other time slices or geographic areas derived from correlative models are compromised by uncertainty (Rocchini et al., 2011). Unfortunately, Turkozan et al. (2021) did not account for such uncertainties.

To examine the impact of extrapolation on their results, we used multivariate environmental similarity surface (MESS) analyses (Elith et al., 2010). MESS analyses identify areas where one or more predictor variables experience conditions beyond the respective calibration range and, thus, are compromised by extrapolation (cf. Elith et al., 2010). To construct MESS maps, we georeferenced the minimum convex polygons (MCPs) used as model training range by Turkozan et al. (2021) and performed MESS analyses using the packages dismo (Hijmans et al., 2017) and raster (Hijmans, 2020) for Cran R (R Development Core Team, 2020). MESS analyses were computed for each of the ten predictors used by Turkozan et al. (2021) separately, rescaled to 0 (no extrapolation) and 1 (extrapolation) and subsequently summed to show the number of variables affected by extrapolation per geographic region (for R code, see Supplementary Materials).

Our results show that the range estimates of Turkozan et al. (2021) are significantly compromised by extrapolation. This refers to vast areas of the study region, for current conditions as well as reconstructions (mid-Holocene and LGM) across all three used general circulation models (GCMs; Supplementary Materials: Figs. S1-5).

Parenthetically it may be noted that Turkozan et al. (2021) erred when they suggested that factor loadings of a principal component analysis (PCA) have been interpreted erroneously by Javanbakht et al. (2017). In contrast to MaxEnt, the non-parametric multivariate approach used by Javanbakht et al. (2017) requires orthogonal input variables. To ensure orthogonality, input variables are subjected to a PCA prior to modelling (Barros et al., 2016), and the (past) climate reconstructions are projected in the PCA space derived from current climate conditions, resulting in different sets of principal components for each scenario. Thus, Turkozan et al. (2021) apparently misunderstood the matter and misinterpreted data presented by Javanbakht et al. (2017) within the frame of another method (MaxEnt).

In addition to these methodological issues, the study by Turkozan et al. (2021) contains additional flaws. For instance, Turkozan et al. (2021) state in their Abstract that "Since the LGM, we hypothesise that the ranges of lineages have either expanded (T. g. ibera), contracted (T. g. zarudnyi) or remained stable (T. g. terrestris), and for other two taxa (T. g. armeniaca and T. g. buxtoni) the pattern remains unclear." This contradicts the Discussion section (p. 15), where the authors state that "the distribution model of $T$. graeca clades in the present work are in line with the classical glacial range contraction and interglacial range expansion model (Stewart et al., 2010) except the zarudyni [sic!] clade which contracted during the interglacial period." However, the authors did not present any convincing evidence for the latter statement. Turkozan et al. (2021: p. 15) explained that their "analysis supports multiple potential refugia during LGM, namely Caucasus, Anatolia, and Balkans" and that "this is in line with the concept that temperate adapted taxa are confined to southern refugia (Stewart et al., 2010)." Stewart et al. (2010) define refugia as the geographical regions that correspond to the species' maximally contracted geographical range during a glacial period. This is in line with the general understanding of glacial refugia (e.g., Hewitt, 2000; Joger et al., 2007; Schmitt, 2007). Neither Javanbakht et al. (2017) nor Turkozan et al. (2021) inferred massive range restrictions during the last glacial cycle. Instead, it seems that climatically suitable space for T. g. armeniaca, T. g. buxtoni and $T$. g. zarudnyi experienced only slight shifts since the LGM, what contrasts with the massive Holocene range expansions of thermophilic species in more northern latitudes (Hewitt, 2000; Joger et al., 2007; Schmitt et al., 2007) and the classical refugia model. This situation has been discussed in detail in Javanbakht et al. (2017) and the interested reader is referred to this publication.

Another misinterpretation of the results of Javanbakht et al. (2017) concerns bioclimatic variables shaping the distribution of $T$. graeca. Turkozan et al. (2021: p. 15) state that Javanbakht et al. (2017) ignored temperature-related factors delimiting the species distribution. However, Javanbakht et al. (2017: p. 635) stated that, besides precipitation as the main variable, "other environmental variables shaping the distribution of tortoises in Iran and Transcaucasia are the seasonal variation in temperature expressed as 'temperature seasonality' and 'annual temperature range'" [and that] "seasonal temperature variation seems to be a limiting factor for tortoises in the Middle East, since this region is characterised by a continental climate with hot summers 
and cold winters. Hence, the combination of precipitation and high temperature seasonality appear to shape the distributional pattern of T. graeca in the eastern part of its range."

Our Short Note revealed that the results of Turkozan et al. (2021) are compromised by misconceptions and misunderstandings. Therefore, they should be interpreted with caution.

\section{REFERENCES}

Barros, C., Thuiller, W., Georges, D., Boulangeat, I. \& Münkemüller, T. (2016). N-dimensional hypervolumes to study stability of complex ecosystems. Ecology Letters 19(7), 729-742. DOI: 10.1111/ele.12617.

Blonder, B. (2018). Hypervolume concepts in niche- and traitbased ecology. Ecography 41(9), 1441-1455. DOI: 10.1111/ ecog.03187.

Blonder, B., Lamanna, C., Violle, C. \& Enquist, B.J. (2014). The $n$-dimensional hypervolume. Global Ecology and Biogeography 23(5), 595-609. DOI: 10.1111/geb.12146.

Ceballos, G., Ehrlich, P.R. \& Raven, P.H. (2020). Vertebrates on the brink as indicators of biological annihilation and the sixth mass extinction. Proceedings of the National Academy of Sciences of the USA 117(24), 13596-13602. DOI: 10.1073/ pnas.1922686117.

Elith, J. \& Leathwick, J.R. (2009). Species distribution models: Ecological explanation and prediction across space and time. Annual Review of Ecology, Evolution, and Systematics 40, 677-697. DOI: 10.1146/annurev.ecolsys.110308.120159.

Elith, J., Kearney, M. \& Phillips, S. (2010). The art of modelling range-shifting species. Methods in Ecology and Evolution 1(4), 330-342. DOI: 10.1111/j.2041-210X.2010.00036.x.

Engler, J.O. \& Rödder, D. (2012). Disentangling interpolation and extrapolation uncertainties in ecological niche models: A novel visualization technique for the spatial variation of predictor variable colinearity. Biodiversity Informatics 8 , 30-40. DOI: 10.17161/bi.v8i1.4326.

Hewitt, G. (2000). The genetic legacy of the Quaternary ice ages. Nature 405(6789), 907-913. DOI: 10.1038/35016000.

Hijmans, R.J. (2020). Package 'raster'. Downloaded on 1 December 2020. https://cran.r-project.org/web/packages/ raster/index.html.

Hijmans, R.J., Phillips, S., Leathwick, J., Elith, J. \& Hijmans, M.R.J. (2017). Package 'dismo'. Circles 9, 1-68.

Ihlow, F., Dambach, J., Engler, J.O., Flecks, M., Hartmann, T., Rajaei, H. \& Rödder, D. (2012). On the brink of extinction? How climate change may affect global chelonian species richness and distribution. Global Change Biology 18(5), 1520-1530. DOI: 10.1111/j.1365-2486.2011.02623.x.

Javanbakht, H., Ihlow, F., Jablonski, D., Široký, P., Fritz, U., Rödder, D., Sharifi, M. \& Mikulíček, P. (2017). Genetic diversity and Quaternary range dynamics in Iranian and Transcaucasian tortoises. Biological Journal of the Linnean Society 121(3), 627-640. DOI: 10.1093/biolinnean/blx001.

Joger, U., Fritz, U., Guicking, D., Kalyabina-Hauf, S., Nagy, Z.T. \& Wink, M. (2007). Phylogeography of western Palaearctic reptiles - Spatial and temporal speciation patterns. Zoologischer Anzeiger 246(4), 293-313. DOI: 10.1016/j. jcz.2007.09.002.
Owens, H.L., Campbell, L.P., Dornak, L.L., Saupe, E.E., Barve, N., Soberón, J., Ingenloff, K., Lira-Noriega, A., Hensz, C.M., Myers, C.E. \& Peterson, A.T. (2013). Constraints on interpretation of ecological niche models by limited environmental ranges on calibration areas. Ecological Modelling 263, 10-18. DOI: 10.1016/j.ecolmodel.2013.04.011.

Phillips, S.J., Anderson, R.P. \& Schapire, R.E. (2006). Maximum entropy modeling of species geographic distributions. Ecological Modelling 190(3-4), 231-259. DOI: 10.1016/j. ecolmodel.2005.03.026.

Phillips, S.J. \& Dudík, M. (2008). Modeling of species distributions with Maxent: New extensions and a comprehensive evaluation. Ecography 31(2), 161-175. DOI: 10.1111/j.0906-7590.2008.5203.x.

R Development Core Team. (2020). R: A Language and Environment for Statistical Computing. Downloaded 20 December 2020. https://www.R-project.org/.

Rocchini, D., Hortal, J., Lengyel, S., Lobo, J.M., Jiménez-Valverde, A., Ricotta, C., Bacaro, G. \& Chiarucci, A. (2011). Accounting for uncertainty when mapping species distributions: The need for maps of ignorance. Progress in Physical Geography 35(2), 211-226. DOI: 10.1177/0309133311399491.

Rödder, D., Lawing, A.M., Flecks, M., Ahmadzadeh, F., Dambach, J., Engler, J.O., Habel, J.C., Hartmann, T., Hörnes, D., Ihlow, F., Schidelko, K., Stiels, D. \& Polly, P.D. (2013). Evaluating the significance of paleophylogeographic species distribution models in reconstructing Quaternary range-shifts of Nearctic chelonians. PLoS One 8(10), e72855. DOI: 10.1371/ journal.pone.0072855.

Schmitt, T. (2007). Molecular biogeography of Europe: Pleistocene cycles and postglacial trends. Frontiers in Zoology 4(1), 11. DOI: 10.1186/1742-9994-4-11.

Stewart, J.R., Lister, A.M., Barnes, I. \& Dalén, L. (2010). Refugia revisited: Individualistic responses of species in space and time. Proceedings of the Royal Society B 277(1682), 661671. DOI: 10.1098/rspb.2009.1272.

TTWG [Turtle Taxonomy Working Group]. (2017). Turtles of the World. Annotated Checklist and Atlas of Taxonomy, Synonymy, Distribution, and Conservation Status (8th Ed.). New York, NY, and Lunenburg, MA, USA (Chelonian Research Monographs, 7). DOI: 10.3854/crm.7.checklist. atlas.v8.2017.

Turkozan, O., Karacaoğlu, Ç. \& Parham, J.F. (2021). Reconstructions of the past distribution of Testudo graeca mitochondrial lineages in the Middle East and Transcaucasia support multiple refugia since the Last Glacial Maximum. Herpetological Journal 31(1), 10-17. DOI: 10.33256/31.1.1017.

VanDerWal, J., Shoo, L.P., Graham, C. \& Williams, S.E. (2009). Selecting pseudo-absence data for presence-only distribution modeling: How far should you stray from what you know? Ecological Modelling 220(4), 589-594. DOI: 10.1016/j.ecolmodel.2008.11.010. 